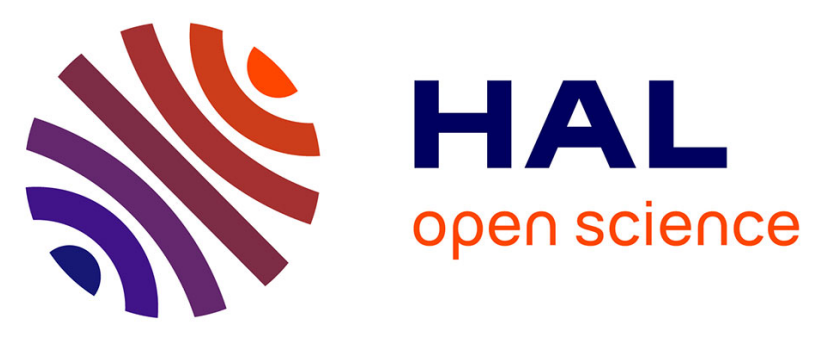

\title{
A New Electrolyte Formulation for Securing High Temperature Cycling and Storage Performances of Na-Ion Batteries
}

\author{
Guochun Yan, Kyle Reeves, Dominique Foix, Zhujie Li, Claudio Cometto, \\ Mariyappan Sathiya, Mathieu Salanne, Jean-marie Tarascon
}

\section{To cite this version:}

Guochun Yan, Kyle Reeves, Dominique Foix, Zhujie Li, Claudio Cometto, et al.. A New Electrolyte Formulation for Securing High Temperature Cycling and Storage Performances of Na-Ion Batteries. Advanced Energy Materials, 2019, 9 (41), pp.1901431. 10.1002/aenm.201901431 • hal-03028024

\section{HAL Id: hal-03028024 https://hal.science/hal-03028024}

Submitted on 27 Nov 2020

HAL is a multi-disciplinary open access archive for the deposit and dissemination of scientific research documents, whether they are published or not. The documents may come from teaching and research institutions in France or abroad, or from public or private research centers.
L'archive ouverte pluridisciplinaire HAL, est destinée au dépôt et à la diffusion de documents scientifiques de niveau recherche, publiés ou non, émanant des établissements d'enseignement et de recherche français ou étrangers, des laboratoires publics ou privés. 
A new electrolyte formulation for securing high temperature cycling and storage performances of $\mathrm{Na}$-ion batteries

\author{
Guochun Yan ${ }^{1,2,+}$, Kyle Reeves ${ }^{2,3}$, Dominique Foix ${ }^{2,5}$, Zhujie Li ${ }^{2,3,4}$, Claudio Cometto ${ }^{1,2}$, Sathiya \\ Mariyappan $^{1,2}$, Mathieu Salanne ${ }^{2,3,4}$, Jean-Marie Tarascon ${ }^{1,2,3, *}$ \\ ${ }^{1}$ Chimie du Solide-Energie, UMR 8260, Collège de France, 75231 Paris Cedex 05, France \\ ${ }^{2}$ Réseau sur le Stockage Electrochimique de l'Energie (RS2E), FR CNRS 3459, Amiens 80039, France \\ ${ }^{3}$ Sorbonne Université, CNRS, 75005 Paris, France \\ ${ }^{4}$ Maison de la Simulation, CEA, CNRS, Université Paris-Saclay, F-91191 Gif-sur-Yvette, France \\ ${ }^{5}$ IPREM/ECP (UMR 5254), Université de Pau, 2 Avenue Pierre Angot, 64053 Pau Cedex 9, France
}

\title{
Abstract
}

The Na-ion battery is recognized as a possible alternative to Li-ion battery for applications where power and cost override energy density performances. However, the increasing instability of their electrolyte with temperature is still problematic. Thus, a central question remains how to design Na-based electrolytes. Here, we report discovery of a Na-based electrolyte formulation which enlists four additives (vinylene carbonate (VC), succinonitrile (SN), 1, 3-propane sultone (PS) and sodium difluoro(oxalate)borate (NaODFB) in proper quantities that synergistically combined their positive attributes to lead a stable solid electrolyte interphase (SEI) at both negative and positive electrodes surface at $55{ }^{\circ} \mathrm{C}$. Moreover, we rationalized the role of each additive that consists in producing specific $\mathrm{NaF}$ coatings, thin elastomers, sulfate-based deposits and so on via combined impedance (EIS) and X-ray photoelectron spectroscopy (XPS). We demonstrated that empirical electrolyte design rules previously established for Li-ion technology together with theoretical guidance is a vital strategy in the quest for better Na-based electrolytes that can be extended to other chemistries. Overall, this finding, which we implement to practical 18650 cells, widens the route to the rapid development of the Na-ion technology based on the $\mathrm{Na}_{3} \mathrm{~V}_{2}\left(\mathrm{PO}_{4}\right)_{2} \mathrm{~F}_{3} / \mathrm{C}$ chemistry.

\footnotetext{
'Present address: School of Metallurgy and Environment, Central South University, 410083 Changsha, China
} 


\section{Introduction}

Due to its success in the domain of power electronics, the lithium-ion battery is currently being considered as the most promising technology for electric vehicle propulsion and stands as a serious contender for grid applications. ${ }^{[1]}$ However, the implementation of a lithium-based technology on a large scale faces an important challenge linked to the lithium availability and cost. ${ }^{[2]}$ In anticipation of such a scenario, new sustainable chemistries have been studied, and the most appealing alternative is to use sodium instead of lithium. Sodium resources are in principle unlimited and sodium is very easy to recuperate. Mindful of these considerations, the sodium-ion battery has regained intensive interest over the last decade with the emergence of new materials for both positive and negative electrodes. ${ }^{[3]}$ Practically speaking, efforts are presently parted between two technologies, both using carbon negative electrodes, but differing by the nature of positive electrode which can be either layered $\left(\mathrm{Na}_{2 / 3} \mathrm{Fe}_{1 / 2} \mathrm{Mn}_{1 / 2} \mathrm{O}_{2}\right)^{[4]}$ or polyanionic $\left(\mathrm{Na}_{3} \mathrm{~V}_{2}\left(\mathrm{PO}_{4}\right)_{2} \mathrm{~F}_{3}\right)^{[5]}$ compounds, denoted hereafter as NFM and NVPF, respectively. While the former shows inherently larger capacity in the half-cell configuration, it suffers from limited power rate and low energy density due to its low redox voltage as compared to the NVPFbased sodium-ion systems. That has sealed, our early decision to opt for the development of sodium-ion NVPF/C technology using $1 \mathrm{M} \mathrm{NaPF}_{6}$ in a 1: 1 (by volume) mixture of ethylene carbonate (EC) - dimethyl carbonate (DMC) as electrolyte. This approach has recently led to the assembly 18650 prototypes showing excellent long-term cycling performance (> 3000 cycles) and high-power rate capabilities at room temperature ${ }^{[6]}$.

Further probing this technology, we found that cycling together with self-discharge performances of the NVPF/C sodium-ion cells were rapidly degrading when increasing the temperature above $55^{\circ} \mathrm{C}$ when linear carbonates (DMC, ethyl methyl carbonate (EMC), diethyl carbonate (DEC)) were part of the electrolyte composition. Such deterioration was shown through complementary operando ultraviolet (UV) spectroscopy and cyclic voltammetry (CV) analytical techniques to be nested in the existence of a shuttle mechanism. It enlists the formation of parasitic products at the negative electrode due to the reduction of linear carbonates that pass into solution and move to the positive electrode, hence accounting for the overall poor performance of the cell at high temperatures. ${ }^{[7]}$ Such a finding came as a surprise, since 
commercial lithium-ion cells are using linear carbonates based electrolytes without showing such limitations.

The problematic nature of electrolyte decomposition/instability is simply nested on the fact that $\mathrm{Li}(\mathrm{Na})$-ion batteries operate beyond the thermodynamic stability voltage domain of the electrolytes, which is related to the positions of the highest occupied molecular orbitals (HOMO) and lowest unoccupied molecular orbitals (LUMO). In most cases, the redox potentials of the positive and negative electrodes fall outside this stability domain, hence triggering the formation of the solid electrolyte interphase (SEI) whose growth is kinetically governed. ${ }^{[8]}$ Mastering/controlling parasitic reactions that lead to the formation of the SEI is then essential to enhance battery's cycle life, rate capability and durability. Ideally, the SEI should have a uniform morphology, a good adhesion to the electrode together with a high ionic conductivity for fast charge transfer while being mechanically strong, insoluble and stable upon cycling. Although the sodium-ion technology looks similar in its principle to lithium-ion batteries, the milder acidic character of $\mathrm{Na}^{+}$results in a different (comparatively higher) solubility of the formed SEI components (sodium-based organic/inorganic products) as compared to their lithium counterparts. ${ }^{[9]}$ Both the parasitic reactions and solubility of SEI components are more pronounced at high temperatures $\left(55^{\circ} \mathrm{C}\right)$, hence necessitating the impetus to design a new electrolyte formulation for enhancing the performances of sodium-ion cells operating above $55^{\circ} \mathrm{C}$.

A common way to reach such an idealized situation is rooted in the use of film-forming electrolyte additives that are added in small amounts while not affecting the bulk electrolyte properties. Many years of experience have taught the need to have several additives that can operate in synergy to provide an optimal SEI, hence the countless amounts of papers and patents on film-forming additives. ${ }^{[10]}$ This longstanding effort has yielded a colossal amount of data out of which one can extract several robust experimental trends/rules for selecting the proper additives that work in synergy. Among them is for instance the synergetic effect between triple-bonded and double-bonded moieties early demonstrated by Abe and recently used by J. Dahn to enable the proper functioning of Li-NMC/C lithium-ion cells up to 4.6 V or the well-recognized beneficial effect of sulfur-containing electrolyte additives, which upon reduction to sulfites and sulfate species, lead to the robustness of the SEI film formed at the negative electrode. ${ }^{[11]}$ 
computational calculations, we report here a novel electrolyte formulation relying on the synergy between additives which enables high interfacial stability at $55{ }^{\circ} \mathrm{C}$, hence setting a new milestone in the practical development of sodium-ion batteries based on the NVPF/C chemistry. Although well aware of the subtle differences between the lithium and sodium-ion chemistries in terms of electrolytes, we have used as starting point of inspiration the same trends pertaining to the lithium-ion technology for selecting additives to confection and optimize an electrolyte for sodium-ion batteries. This approach led us to the identification of an electrolyte formulation $\left(1 \mathrm{M} \mathrm{NaPF}_{6}\right.$ in $\mathrm{EC}-\mathrm{PC}+$ a series of additives) that enables NVPF/C sodium-ion cells to operate at $55{ }^{\circ} \mathrm{C}$ with limited self-discharge and good cycling stability as none of the reported electrolytes could have done it. Such a finding was rationalized via complementary Electrochemical Impedance Spectroscopy (EIS) and X-ray photoelectron spectroscopy (XPS).

Finally, we have extended our studies with a combination of molecular dynamics (MD) and DFT calculations, to ascertain the validity of additive mixtures with the hope to demonstrate their synergy. ${ }^{[12]}$ Such calculations can estimate the thermodynamic potential domain of electrolytes with and without additives, hence it is expected to provide guidance in identifying a proper electrolyte formulation. Our results show that these calculations are still limited to qualitative understanding only, and they cannot be used to probe a potential synergetic effect. Hence, despite the advances made, we still need to resort to trial and error approaches to identify the best electrolyte additives for new battery chemistries. For reasons of clarity we will first present the electrochemical performances of each additive prior to consider their synergetic effects.

\section{Results and discussion}

Mindful of our early results regarding the inadequacy of having linear carbonate such as DMC in the electrolyte, ${ }^{[7]}$ we opted for using a $1 \mathrm{M} \mathrm{NaPF}_{6} \mathrm{EC}-\mathrm{PC}$ (50:50 by volume) electrolyte solution as our "mother" electrolyte. Additives were selected based on both chemical considerations and DFT calculations aiming at determining their LUMO and HOMO. From the chemical survey, our choice boildown to mainly four additives for reasons listed below. Lithium difluoro(oxalate)borate (LiODFB) was used in the lithium-ion technology because of its proven ability to enhance the SEI stability via the growth of an 
SEl rich in LiF nanoparticles. ${ }^{[13]}$ We thus decided to use the equivalent NaODFB salt hoping that it will behave alike in the Li-ion batteries. Getting further inspiration from the lithium-ion technology and the positive synergism between triple and double bonds we selected a combination of succinonitrile (SN) and vinylene carbonate $(\mathrm{VC})$ additives with the former having the $(-\mathrm{C} \equiv \mathrm{N})$ triple bond and the latter the $(\mathrm{C}=\mathrm{C})$ double bond. The choice for SN was additionally rooted in its strong nucleophilic character with the hope that it could, via surface interaction, stabilize the high oxidation state of $\mathrm{V}^{5+}$, hence minimizing high voltage parasitic reactions associated to the electrolyte. ${ }^{[12 a, 14]}$ Lastly, bearing in mind the evidence of radical species formed during the formation of the SEI and its evolution upon cycling in NVPF/C Na-ion batteries as previously reported we decided to test the effect on the high-temperature performances of the 1,3-propane sultone (PS), since this molecule is known to trap radicals inside the electrolyte, thus forming reduced sulfite or sulfate inorganic compounds. ${ }^{[15]}$

To validate this choice, we determine the position of the LUMO-HOMO levels of each of these additives via DFT calculations bearing in mind that the best film-forming additives are the ones with HOMO- and LUMO falling within those of the electrolyte solvent molecules. As shown in supplementary figure 1, the most promising additives based on this criterion are the ODFB ${ }^{-}$anion and the VC. Albeit to a lesser extent, the two levels of the PS and the LUMO of the SN also stand between the ones of the EC and the PC, which suggests that these molecules may also react at the interfaces to form a SEl. Yet, while providing guidance regarding the selection of individual molecules, such calculations cannot be blindly used for reasons described elsewhere. ${ }^{[16]}$ Moreover, such calculations fail to predict the synergism between additives, the reason why we have utilized systematic trial-and-error approach to identify the best electrolyte configuration.

All the aforementioned additives were obtained from Sigma-Aldrich and dried under molecular sieves prior to be used with the exception of NaODFB that is not commercialized. Thus NaODFB was homemade according to the following reaction via a process derived from the references ${ }^{[17]}$, which consists in $\mathrm{BF}_{3}$ reacting with $\mathrm{Na}_{2} \mathrm{C}_{2} \mathrm{O}_{4}$ in a 1,2-dimethoxyethane (DME) solution in 1:1 ratio, at $80^{\circ} \mathrm{C}$ under argon for 24 hours. NaODFB is then recovered by the removal of DME, dried under vacuum and stored in 
132

133

an argon glove box prior to be identified as single phase by FTIR, XRD, ${ }^{19} \mathrm{~F}$ and ${ }^{23} \mathrm{Na}$ NMR. (see supplementary figures 2-4)

For reasons of clarity, we will first present the effects of each additive (NaODFB, PS, VC and SN) separately (for amounts ranging from 1 to $5 \%$ ) on the electrochemical performances of NVPF/C sodiumion coin cells using $1 \mathrm{M}-\mathrm{NaPF}_{6}$ in $\mathrm{EC}-\mathrm{PC}$ as mother electrolyte. Measurements were performed at $55^{\circ} \mathrm{C}$, if not otherwise specified, according to an experimental protocol (Fig. 1b) that consists in cycling the cell 10 times prior to performing a one week self-discharge at $100 \%$ state of charge $(\sim 4.3 \mathrm{~V})$ and cycling again at $\mathrm{C} / 10$ rate $\left(1 \mathrm{C}=128 \mathrm{~mA} \mathrm{~g}^{-1}\right)$. We solely report the data that has been duplicated twice. $55^{\circ} \mathrm{C}$ rather than room temperature testing was privileged to enhance the effect of additives onto the electrolyte stability since degradation is usually related to kinetically-driven parasitic reactions. We use four figures of merits to compare the electrolytes: i) the percentage of capacity retention before and after one week of selfdischarge defined as $Q_{11 \text { th }}$-dis $/ Q_{11 \text { th }}$-cha (discharge capacity divided by the charge capacity of the $11^{\text {th }}$ cycle), ii) the percentage of recovered capacity determined as $Q_{12 t h}$-dis/ $Q_{11 \text { th }}$-cha, iii) the capacity retention and iv) the cell resistance evolution upon cycling.

Figure 1 compares the performances of each additive separately. From the first cycle voltagecapacity curves (Fig. 1a), it can be deduced that the irreversible capacity between the first charge and discharge is smaller for $\mathrm{VC}$ and NaODFB implying that such additives can partially mitigate the decomposition of the electrolyte at the carbon electrode by forming an effective SEI layer, as expected based on the DFT calculations. In contrast, the irreversibility is the largest for SN, suggesting its decomposition at the negative electrode consuming the sodium ions. Finally, PS has no effect on the irreversible capacity. The corresponding derivative curves, which we plotted from 2 to $2.8 \mathrm{~V}$ for better bring out anomalies (Fig. 1a, inset), reveal a sharp peak that is observed solely for electrolyte having NaODFB. This peak that appears at $2.1 \mathrm{~V}$ (in NVPF/ HC full cells) is due to the reduction of NaODFB at hard carbon electrode as confirmed by the cyclic voltammetry experiments (supplementary figures 5). In contrast, no distinct peaks are observed for VC, SN, or PS additives. The absence of a reduction peak for VC contrasts from its commonly observed behavior in lithium-based electrolytes, ${ }^{[18]}$ suggesting a different reduction mechanism. Cycling-wise (Fig. 1c) NaODFB stands out among the other additives. 
Fig. $1 \mathrm{~b}$ shows the effect of various additives on the percentage of capacity retention in the self-

160

161

162

163

164

165

166

167

168

169

170

171

172

173

174

175

176

177

178

179

180

181

182

discharge cycle $\left(C_{S}\right)$ and capacity recovered in the recovery cycle $\left(C_{R}\right)$ by holding the cells at $55^{\circ} \mathrm{C}$ for one week in self-discharge. $C_{S}$ (inset left) and $C_{R}$ (inset right) values range from 79 to $89.5 \%$, and 91.6 to 95.4 $\%$ respectively. Such values bear interesting information if we keep in mind that the self-discharge is the result of cumulative side reactions taking place at the anode and cathode. The feasibility to recover a major fraction of the capacity implies that part of the parasitic reactions is reversible. Through our previous study we have demonstrated that the oxidation reaction taking place at the positive electrode and leading to the reduction of NVPF was nearly fully reversible, in contrast to the parasitic reaction taking place at the carbon electrode. ${ }^{[7 b]}$ Bearing this in mind, we can deduce that the best additives, selfdischarge wise, are the ones showing both high $C_{S}$ and $C_{R}$ values, which is with minimum amounts of side reactions at the anode and cathode, respectively. On these metrics, both NaODFB and PS perform equally well while $\mathrm{SN}$ is the worst candidate, consistent with the fact that it leads to the highest initial irreversible capacity. Lastly, we compared the effect of these additives on the cell resistance and we observed a distinct increase in resistance with cycling for the NaODFB and VC (Fig. 1d), which does not come as a surprise since both of these additives are prone, as disclosed from the DFT calculation (see supplementary figure 1), to react at the interfaces to form a SEI.

Altogether, these results indicate that neither of the additives provides sufficient performances for practical applications, but NaODFB stands out as it displays the best figures of merit for three criteria, namely the initial reversible capacity, self-discharge performances and capacity retention. Besides identifying an additive it is also of paramount importance to determine its optimum concentration. From a survey of various electrolytes with increasing percentage content of NaODFB, we identified $\sim 0.5 \%$ as the optimum content. Greater contents were experimented to lead to unpractical oxidation of the ODFB anion at the NVPF electrode (see supplementary figure 6). Hence, from now on NaODFB in a content of $0.5 \%$ by weight will be used as additive in all new explored electrolytes formulations.

Our second experimental iteration aims to lower the cell resistance increase by using NaODFB (Fig. 1d). To approach this issue we first surveyed the effects of PS and VC additives on the performances of 1M EC-PC electrolyte solutions containing $0.5 \%$ of NaODFB. We found the addition of $3 \%$ PS gives lower 

cell resistance (Fig. 2c), a good capacity retention (Fig. 2b) but a large self-discharge (27.9\%), while the addition of $5 \%$ VC leads to poor capacity retention (Fig. 2b) in agreement with the progressive increase in the cell resistance (Fig. 2c). This does not come as a surprise since an increase in cell resistance was also observed when VC is used as a single additive (Fig. 1).

At this stage we extended this work to the exploration of the double-triple bond synergy between additives. A third additive $S N(-C \equiv N)$ was then added to the VC $(C=C)$-based electrolyte but without noticeable improvements. In contrast, low self- discharge and limited resistance increase were observed with the addition of $1 \%$ SN to the mixture of 3\% PS and $0.5 \%$ NaODFB (Fig. 2a and Fig. 2c). The negative results observed with VC were somewhat surprising owing the well admitted success of VC with the Li-ion technology. Indeed, by its nature VC enhances the stability of the SEI forming at negative electrode via an electrochemically-driven polymerization, provided that it is not used in excess. Otherwise, it can be detrimental to the cell performance due to its copious oxidative decomposition at the positive electrode that generates a cell impedance increase. ${ }^{40}$ Thus, our last iteration has consisted of adding various content of VC to our best three components mixture so far isolated and exploring its electrochemical performance by varying its content from $0,1,2,3$ and $5 \%$. The results summarized in Fig. 3 indicate that the electrolyte consisting of $1 \mathrm{M} \mathrm{NaPF}_{6}$ in $\mathrm{EC}-\mathrm{PC}+3 \% \mathrm{PS}+0.5 \% \mathrm{NaDOFB}+1 \% \mathrm{SN}+3 \% \mathrm{VC}$, and denoted hereafter as magic $A$, displays outstanding performances in terms of $C_{R}(99 \%)$ and $C_{S}(89.5 \%)$ with additionally the highest capacity retention (99\%) after 60 cycles while having the lowest cell resistance of all the sodium-based electrolytes tried so far. It is worth mentioning that for VC contents exceeding $3 \%$, alike for the Li-ion technology, a detrimental effect on the cell impedance is observed. ${ }^{[19]}$

Although this new electrolyte cocktail finding has been achieved through a trial and error approach, we must understand and rationalize the underlying science. Specifically, it is important to characterize the mechanisms by which this electrolyte leads to highly performing SEI. To provide insights into this issue we decided to conduct complementary EIS and XPS experiments since these two techniques allow probing interfacial phenomena.

For the EIS experiments, another set of NVPF/C sodium-ion coin cells were assembled with either 1M $\mathrm{NaPF}_{6}$ in EC-PC or magic $\mathrm{A}$ as the electrolyte. As before, the tests were performed at $55^{\circ} \mathrm{C}$ with the cells 
having been activated by carrying out 10 cycles prior to allowing each cell to self-discharge for one week and ultimately cycling it back afterwards. EIS spectra were collected at the end of discharge after $1^{\text {st }}, 10^{\text {th }}$, $11^{\text {th }}$ and $61^{\text {st }}$ cycle for both electrolytes and the Nyquist spectra are reported in Fig. 4a for EC-PC and Fig. $4 \mathrm{~b}$ for magic $\mathrm{A}$. The data is also reported for the $62^{\text {nd }}$ cycle when the cell was cooled down to $25^{\circ} \mathrm{C}$; they all show a similar shape with a depressed semi-circle covering high frequency (interphase contacts, surface film) and high-medium frequency (charge transfer) phenomena with a low frequency Warburg tail restricted to solid phase $\mathrm{Na}^{+}$diffusion. The trends for variations of $R_{s f}$ (surface film resistance) and $R_{c t}$ (charge transfer resistance) vs. state of cycling for both electrolytes are deduced by fitting with the equivalent circuit model shown in Fig. $4 \mathrm{~d}$ and reported in Fig. $4 \mathrm{c}$ and e, respectively. Whatever the electrolyte, $R_{s f}$ continuously increases upon cycling, but at a lower rate for magic $A$, indicative of the formation of a thinner SEl forming layer. Note that after the $61^{\text {st }}$ cycle, $R_{s f}$ for EC-PC electrolyte is nearly twice larger than that for magic A when cooling down the cell from $55^{\circ} \mathrm{C}$ to $25^{\circ} \mathrm{C}$. Interestingly, no change in $R_{s f}$ is observed when the cell based on magic $A$ is cycled back to $25^{\circ} \mathrm{C}$ as opposed to a noticeable increase for EC-PC suggesting the presence of soluble species (alkyl-carbonates, alkyl-oxides) that might precipitate upon returning to room temperature. For the sake of completeness, similar measurements were done for an EC-DMC electrolyte for which we had previously demonstrated the presence of such species in solution and found an increase of nearly $45 \%$ in the cell impedance (see supplementary figure 7).

Turning to the charge transfer resistance (Fig. 4e), the same trend is observed with an even more pronounced effect of magic $A$ since $R_{c t}$ is three times smaller after the $61^{\text {st }}$ cycle for magic $A$ as compared to the mother electrolyte. Moreover, the similar trend followed by both $R_{s f}$ and $R_{c t}$ is linked to the fact that a growth of the SEI (increase in $R_{s f}$ ) will lead to poor ion transport that is reflected by an increase of the charge transfer, hence $R_{c t}$. As previously noted, returning to room temperature does not produce any changes in $\mathrm{R}_{\mathrm{ct}}$ for magic $\mathrm{A}$ as opposed to a $30 \%$ increase for the EC-PC electrolyte, which simply translates by a prominent increase in the cell polarization between $55^{\circ} \mathrm{C}$ and $25^{\circ} \mathrm{C}$. Altogether, these results nicely support the positive attributes of magic A over the EC-PC electrolyte that is rooted in a lower growing rate 
of the SEI forming film, but did not bring any clues about its composition, hence limiting chances to know how it forms.

To grasp more insights into the composition of the SEI films formed at both negative and positive electrodes through our $55^{\circ} \mathrm{C}$ testing protocol, we embarked into the study of electrodes recovered after the $1^{\text {st }}, 10^{\text {th }}, 11^{\text {th }}$ and $61^{\text {st }}$ by means of XPS. Once the targeted cycling was over, the cells were opened in an argon glove box, washed with DMC to remove the residual electrolyte, and dried prior to being packed into a hermetically sealed aluminum plastic bag for transportation. To prevent any sample exposure to moisture/air on the analysis site, the NVPF or carbon electrode samples were removed from the plastic bag within a glove box directly connected to the XPS spectrometer. The $C_{1 s}, O_{1 s}, F_{1 s}, P_{2 p}$ and $S_{2 p}$ spectra were collected for each NVPF and C samples recovered from the aforementioned NVPF/C cells. For reasons of conciseness, we solely report the data for the $C_{1 s}$ (NVPF), $O_{1 s}(N V P F), F_{1 s}(H C)$, and $P_{2 p}(H C)$ core spectra that show the most prominent changes in Fig. 5 (other results are shown in supplementary figures 8-10).

We first report the $\mathrm{C}_{1 \mathrm{~s}}$ and $\mathrm{O}_{1 \mathrm{~s}}$ spectra for NVPF in Fig. $5 \mathrm{a}$ and Fig. $5 \mathrm{~b}$ respectively, for both mother electrolyte (EC-PC) and magic A electrolyte (left and right in each panel) with underneath the quantitative variation of each identified components as deduced from analysis of the core spectra. The $C_{1 s}$ spectrum for EC-PC shows signals pertaining to conductive carbon black and PVdF binders. By integration of these signals we could plot their variation as a function of the cycling number and observe noticeable decrease (Fig. 5a bottom left), which implies they are masked by the growth of a cathode electrolyte interface (CEI). Such an effect still persists with our magic A electrolyte but in a lesser extent as witnessed (Fig. 5a bottom left), implying indirectly the formation of a thinner SEl layer with magic A electrolyte, hence confirming our EIS results. Besides the peaks associated to $\mathrm{C}$ black and PVDF, the C1s core spectra reveals upon cycling the appearance of 3 new peaks located at $285.9 \mathrm{eV}, 287.4 \mathrm{eV}$ and $289.2 \mathrm{eV}$, which can be assigned to $\mathrm{C}-\mathrm{CO}(=\mathrm{O})$ or $\mathrm{C}_{-} \mathrm{CO}_{3}, \mathrm{C}=\mathrm{O}$ or $\mathrm{O}-\mathrm{C}-\mathrm{O}$, and $\mathrm{OC}=\mathrm{O}$ or $\mathrm{CO}_{3}$, respectively. We estimated the variation in amplitude of these signals as a function of cycling and the results are shown in Fig. 5a bottom. Their evolution reveals a rapid increase for the EC-PC electrolyte as opposed to a minor increase for our magic A electrolyte, reminiscent of the formation of a stable CEI. 
Next we explored the $\mathrm{O}_{1 \mathrm{~s}}$ spectra (Fig. $5 \mathrm{~b}$ ), and observed the presence of three signals: two located at $532.2 \mathrm{eV}$ (blue) and $533.6 \mathrm{eV}$ (orange) associated to oxygenated carbon species previously spotted with the $C_{1 s}$ spectra, and another one at $531.4 \mathrm{eV}$ (red) corresponding to the oxygen pertaining to the phosphate group in $\mathrm{Na}_{3} \mathrm{~V}_{2}\left(\mathrm{PO}_{4}\right)_{2} \mathrm{~F}_{3}$. Interestingly, the amplitude of this signal remains constant upon cycling for magic A electrolyte, while it decreases to the expense of the blue and orange ones for the ECPC electrolyte, indicating its copious growth. We also collected the $P_{2 p}$ and $V_{2 p}$ core spectra for cycled NVPF electrodes and further confirm the same trend (see supplementary figure 8). Altogether, these results indicate a much greater stability of the $\mathrm{CEI}$ in magic $\mathrm{A}$ than in EC-PC electrolyte.

To grasp some light on the growth and nature of SEI forming at the negative electrode we next collected the $F_{1 s}$ and $P_{2 p}$ core spectra as shown in Fig. $5 c$ and $5 d$ respectively. From the $F_{1 s}$ spectra, we observe the presence of a peak at $684.6 \mathrm{eV}$ after the $1^{\text {st }}$ cycle that we can ascribed to NaF derived from the decomposition of $\mathrm{NaPF}_{6}$ with its amplitude in EC-PC being more than 10 times larger than the one observed for magic $A$. Moreover, the $C_{1 s}$ core spectra of the carbon electrode for magic $A$ revealed signals located at 290.7, 284.1, 286.6, and $288.8 \mathrm{eV}$ and corresponding to $\mathrm{C}-\mathrm{F}, \mathrm{C}-\mathrm{H}, \mathrm{C}-\mathrm{O}$ and $\mathrm{O}=\mathrm{C}-\mathrm{O}$ functional groups, respectively, with nearly the same groups appearing for the EC-PC electrolyte at the exception of C-F (see supplementary figure 9 and table 1, respectively). Lastly, from the $S_{2 p}$ core spectra (see supplementary figure 10), we could nicely spot the emergence of a signal at $169.0 \mathrm{eV}$ and corresponding to sodium sulfate $\left(\mathrm{Na}_{2} \mathrm{SO}_{4}\right)$ which originated from the decomposition of the PS. These differences clearly indicate the key role played by the additives in controlling the composition and growth of the SEI. electrolyte is composed of large amounts of NaF, alkyl carbonates and PO/POF . The SEl formed in presence of magic A contains nearly the same amount of alkali carbonates or phosphate species, but drastically differs by the lower amount of $\mathrm{NaF}$ and the presence of sulfate and B-based species (see supplementary note 1), hence justifying the use of PS and NaODFB additives. We believe that the positive attribute of the NaODFB additives is similar to what had been previously observed, to form deposits of NaF nanocrystallites forming thin and dense SEI layer (See EDS mapping in supplementary Figure 11). In contrast, the composition of the CEI layer forming at the positive electrode upon cycling mainly consists of 

A as compared to EC-PC electrolyte.

Overall, complementary XPS and EIS measurements have shown that the gained electrochemical performances display by magic A with respect to EC-PC based electrolyte is mainly rooted in the composition of the formed CEI at the NVPF positive and SEI formed at the carbon negative electrodes. We could identify the role played by individual additives NaODFB or PS while having no clues regarding their synergy. Motivated by the added knowledge and efficiency that predicting additive synergies could provide in designing new electrolytes, we next conducted further DFT calculations to shed further light on the effect of the four additives. Indeed, although the results provided in supplementary figure 1 (Figure isolated molecules) are instructive, they were performed on single molecules in a polarizable continuum to represent the solvent and therefore do not account for eventual synergetic interactions between electrolyte and additive molecules. We therefore investigate the entire electrolyte system-with the presence of all additives and an explicit solvent - following the example of Ong et al. ${ }^{[20]}$ In short, DFTbased molecular dynamics (DFT-MD) is used to simulate a $\mathrm{NaPF}_{6}$ electrolyte in EC/PC solvent with the molecular additives at the same concentrations as in experiments in order to more closely examine the electronic structure of individual molecules and relate this to the average reactivity of each molecule. This is done by projecting the density of states (DOS) onto an atomic orbital basis set for ten different atomic configurations spaced 2 picoseconds apart throughout the molecular dynamics simulations. The electrochemical windows were determined by identifying the band edges (HOMO and LUMO) nearest to the computed Fermi energy, as shown on Figure 6.

In agreement with the isolated molecules calculations, we notice that the ODFB anion and the VC are likely to be the more electrochemically active of the additive molecules and to participate in the SEI layer formation at the electrode interface. The condensed phase calculations also show that the PS and SN levels now are aligned with the ones of the solvent, which suggests that this molecules will not react directly but rather in a second step with the newly formed species at the SEI. 
We have reported the design of a new electrolyte formulation $1 \mathrm{~mol} \mathrm{~L}^{-1} \mathrm{NaPF}_{6}$ in $\mathrm{EC}-\mathrm{PC}+0.5 \%$ $\mathrm{NaODFB}+3 \% \mathrm{PS}+1 \% \mathrm{SN}+3 \% \mathrm{VC}$, which we have implemented into the assembly of practical $18650 \mathrm{Na}-$ ion batteries that displays attractive high temperature performances $\left(55^{\circ} \mathrm{C}\right)$ in terms of self-discharge and cycling (Supplementary Figure 12). The search for optimum electrolyte formulation still relies on trial and error approaches, as theory presents limitations that we could not presently account for the synergetic effect between the various additives. Over the years, with the knowledge gained so far, several robust empirical rules have emerged such as for instance the synergy between double and triple bond that we have used herein together with theoretical guidance to identify our best electrolyte formulation. In the near future, let's hope the development of artificial intelligence and robotics lines for rapidly conducting surveys, based on the rich existing data bank, will ingress the battery field and provide robust trends nonidentified yet for selecting additives working in synergy.

Besides the right choice of additives, we have shown that their amounts can be critic. VC is for instance needed to create a stable SEl at the negative electrode but its role in large excess can be counterproductive because of its oxidative decomposition leading to copious deposits at the positive electrode. Equally, we found that amounts of NaODFB > 3\% were leading, because of parasitic decomposition reactions were leading to the growth of thick deposits at the positive electrode and hence a drastic increase in the cell impedance. Through XPS measurement, the role of PS was identified as an enrichment of the SEl layer in sulfates, which is an ingredient known to be beneficial for SEI at least in Li-ion batteries. Lastly, SN in combination with VC appears to be beneficial for the CEI formed at the NVPF positive poor wettability which complicates its use with today's commercial celgard separators. Linear carbonates (DMC, DEC...) or fluorinated ethers (HFE) solvents having good wettability could alleviate this issue, but to the expense of poor high temperature performance as we demonstrated by adding DMC to magic A (Supplementary Figure 13). Obvious improvement of this work calls for the development of an electrolyte with greater wettability while preserving all other figures of merits and also for the use of a fewer number of additives. Although this seems like squaring a circle, this new type of electrolyte is being successfully 
developed and will be the subject of an upcoming paper, which further widens the implications of our findings.

\section{Methods}

Electrolyte preparation. The electrolytes were prepared by dissolving one molar $\mathrm{NaPF}_{6}$ (Stella, Japan) in a battery-grade solvent selected from DMC, PC (BASF, USA), and EC (Mitsubishi chemical, Japan) in the Arfilled glovebox (MBraun MB200B), prior to adding the certain amount of additives that was purchased from Sigma-Aldrich except the home made NaODFB. All solvents and additives were dried by adding molecular sieve (4 $\AA$, Sigma) till their water contents become lower than $10 \mathrm{ppm}$ as determined by KarlFisher titration (Metrohm 899 coulometer).

Electrochemical measurements of NVPF/C cells. $\mathrm{Na}_{3} \mathrm{~V}_{2}\left(\mathrm{PO}_{4}\right)_{2} \mathrm{~F}_{3}\left(\mathrm{NVPF}, 12.0 \mathrm{mg} / \mathrm{cm}^{2}\right)$ tapes were casted by spreading the slurries (NVPF materials, polyvinylidene difluoride and carbon black (the weight ratio is 90:4:6) within $\mathrm{N}$-methyl-2-pyrrolidone) onto Al foil. For the hard carbon (C, $\left.6.0 \mathrm{mg} / \mathrm{cm}^{2}\right)$ tapes, it were casted by spreading the slurries (hard carbon materials, carboxymethyl cellulose and carbon black (the weight ratio is 95:2:3) within deionized water) onto Al foil. All of the electrodes were dried in a Buchi oven under vacuum (lower than $100 \mathrm{mbar}$ ) at 80 degrees for $24 \mathrm{~h}$ prior to be transferred into glovebox. The electrochemical performance of NVPF/C full cells were evaluated in 2032-type coin cells separated by one layer of glass fiber containing $150 \mu \mathrm{L}$ of desired electrolytes. All cells were galvanostatically cycled by using VMP3 or MPG2 potentiostat (Bio-Logic, France) at $55{ }^{\circ} \mathrm{C}$ or $25{ }^{\circ} \mathrm{C}$, and the cycling rates were calculated on the basis of NVPF material $\left(1 \mathrm{C}=128 \mathrm{~mA} \mathrm{~g}^{-1}\right)$. The impedance measurements of the NVPF/C cells after $1^{\text {st }}, 10^{\text {th }}, 11^{\text {th }}$, and $61^{\text {st }}$ in discharged state were conducted by Bio-Logic MPG2 between $100 \mathrm{kHz}$ and $10 \mathrm{mHz}$ with an amplitude of $10 \mathrm{mV}$ signal at $55^{\circ} \mathrm{C}$, and the following $62^{\text {nd }}$ cycle at $25^{\circ} \mathrm{C}$.

X-ray photoelectron spectroscopy measurements. To carry out the XPS measurements, another series of NVPF/C cells were cycled, which were disassembled after the $1^{\text {st }}, 10^{\text {th }}, 11^{\text {th }}$, and $61^{\text {st }}$ cycled in the glove box to recover the NVPF and C electrodes. The obtained electrodes were washed with DMC to remove the residual electrolyte, and dried prior to being packed into a hermetically sealed aluminum plastic bag for transportation. XPS measurements were carried out with a Kratos Axis Ultra spectrometer, using focused monochromatic AI KQ radiation ( $\mathrm{hv}=1486.6 \mathrm{eV}$ ). The XPS spectrometer was directly connected through a 
transfer chamber to an argon dry box, in order to avoid moisture/air exposure of the samples. The analyzed area of the samples was $300 \times 700 \mu \mathrm{m}^{2}$. Peaks were recorded with constant pass energy of $20 \mathrm{eV}$. For the Ag $3 d_{5 / 2}$ line the full width at half-maximum (FWHM) was $0.58 \mathrm{eV}$ under the recording conditions. The pressure in the analysis chamber was around $5 \times 10^{-9}$ mbar. Short acquisition time spectra were recorded before and after each normal experiment to check that the samples did not suffer from degradation during the measurements. The binding energy scale was calibrated using the $\mathrm{C} 1 \mathrm{~s}$ peak at $285.0 \mathrm{eV}$ from the hydrocarbon contamination for the negative electrodes $\mathrm{HC}$, and using the $\mathrm{C}$ 1s peak at $290.9 \mathrm{eV}$ from the PVdF $\mathrm{CF}_{2}$ functions, for the positive electrodes NVPF. Core peaks were analyzed using a nonlinear Shirley-type background ${ }^{[21]}$. The peak positions and areas were optimized by a weighted leastsquares fitting method using $70 \%$ Gaussian, $30 \%$ Lorentzian line shapes. Quantification was performed on the basis of Scofield's relative sensitivity factors ${ }^{[22]}$. The curves fit for core peaks were obtained using a minimum number of components in order to fit the experimental curves.

Single-Molecule Calculations. To compute the HOMO and LUMO levels of EC, PC, VC, PS, SN and ODFBwe perform density functional theory calculations using the CP2K code using the Quickstep algorithm. ${ }^{[23]}$ The relaxed geometry of each molecule was computed using the self-consistent continuum solvation (SCCS) model. ${ }^{[24]}$ The dielectric constant used in the SCCS model was set to 75 , a value that falls roughly halfway between pure EC $\left(90.5\right.$ at $\left.40^{\circ} \mathrm{C}\right)$ and pure PC $\left(65.5\right.$ at $\left.25^{\circ} \mathrm{C}\right) .{ }^{[25]}$ TZV2P-MOLOPT-GTH basis sets ${ }^{[26]}$ were used to construct the Kohn-Sham wave functions, and an energy cutoff of 600 Rydberg was used. A GGA and a hybrid exchange-correlation functional $\left(\mathrm{PBE}^{[27]}\right.$ and $\mathrm{B} 3 \mathrm{LYP}^{[28]}$ respectively) were each tested to compare the computed HOMO and LUMO energies as well as their relative positions between solvent and additive molecules. Goedecker-Teter-Hutter pseudopotentials ${ }^{[29]}$ were used to describe core electrons.

Molecular Dynamics Calculations. i) Classical Molecular Dynamics. Classical MD simulations were performed using LAMMPS code ${ }^{[30]}$ to generate the initial atomic structure for two electrolyte systems. The first electrolyte system without additives contains $38 \mathrm{EC}$ molecules, $30 \mathrm{PC}$ molecules, $7 \mathrm{NaPF}_{6}$ pairs, while the system with additives consists of $38 \mathrm{EC}$ molecules, 30 PC molecules, 3 VC molecules, 2 PS molecules, $1 \mathrm{SN}$ molecule, 1 ODFB anion, $6 \mathrm{PF}_{6}$ anions, and $7 \mathrm{Na}$ cations. The simulation cells were constructed to match the experimental density. An all-atoms model was used for each of the two 
401

402

403

404

405

406

407

408

409

410

411

412

413

414

415

416

417

418

419

420

421

422

423

424

425

426

427

systems, in which the OPLS force field ${ }^{[31]}$ was used for $\mathrm{EC}, \mathrm{PC}$ and $\mathrm{NaPF}_{6}$ molecules. The additive molecules were treated as rigid bodies without considering any intra-molecular interactions. Simulations were conducted in NVT ensemble with Nose- Hoover thermostat. All the systems were equilibrated at $353.15 \mathrm{~K}$ for $15 \mathrm{~ns}$ with a timestep of $1 \mathrm{fs}$. ii) Density Functional Theory. We performed first-principles simulations using density functional theory molecular dynamics. NVT simulations were performed using the PerdewBurke-Ernzerhof (PBE) generalized gradient approximation (GGA). ${ }^{[27]}$ The Quickstep algorithm as implemented in the CP2K code was employed with an energy cutoff of 280 Ryd. Van der Waals corrections were included at the level of DFT-D3. ${ }^{[32]}$ The temperature of the simulation was regulated using canonical sampling through velocity rescaling (CSVR) at a temperature of $328 \mathrm{~K}$. The timestep used was $0.5 \mathrm{fs}$. The simulation cells were identical in dimensions to those used in the classical simulations along with periodic boundary conditions, and the final state of the classical MD was used as a starting configuration. The large size of the simulation cells allows us to perform calculations using only a single $\Gamma$ k-point. Simulations for both systems were run for a total of 20 ps.

To perform the analysis of the projected density of states (DOS) calculations, we identify ten different atomic configurations spaced 2 ps apart throughout the molecular dynamics simulations. For each set of configurations, we used Quantum Espresso code ${ }^{[33]}$ to compute the ground state electron density in order to be projected onto an atomic orbital basis set. We verify the completeness of the atomic orbital basis using the computed spilling parameter. The projection onto the atomic orbital basis $^{[34]}$ has a corresponding spilling parameter between 0.0126 and 0.0127 for the electrolyte system with additives and between 0.0123 and 0.0124 for the electrolyte system, and thus we concluded that this projection can be used to sufficiently construct the density of states. This projection scheme allows us to parse contributions of individual molecules. Therefore, we can approximate the contribution of an individual molecule to the total density of states or alternatively determine the average contribution of a single type of molecule. The electrochemical windows were determined by identifying the band edges (HOMO and LUMO) nearest to the computed Fermi energy. These values for the ten configurations were used to determine the average and standard deviations for $\varepsilon_{\text {номо }}$ and $\varepsilon_{\text {LUMO }}$. Mean values and their standard deviations are reported for HOMO and LUMO levels for each molecule. 
Project 670116-ARPEMA; G. Yan. thanks the National Natural Science Foundation of China for

funding (Grant No. 51804344). The authors thank Arnaud Demortière and Kirill Cherednichenko for

electronic microscopy measurments, and Alix Ladam for assembling the 18650 cells.

[1] a) B. Dunn, H. Kamath, J.-M. Tarascon, Science 2011, 334, 928; b) Z. G. Yang, J. L. Zhang, M. C. W. Kintner-Meyer, X. C. Lu, D. W. Choi, J. P. Lemmon, J. Liu, Chemical Reviews 2011, 111, 3577; c) R. Schmuch, R. Wagner, G. Hörpel, T. Placke, M. Winter, Nature Energy 2018, 3, 267.

[2] a) D. Larcher, J.-M. Tarascon, Nature chemistry 2015, 7, 19; b) C. P. Grey, J. M. Tarascon, Nature Materials 2017, 16, 45.

[3] a) N. Yabuuchi, K. Kubota, M. Dahbi, S. Komaba, Chemical Reviews 2014, 114, 11636; b) B. L. Ellis, L. F. Nazar, Current Opinion in Solid State \& Materials Science 2012, 16, 168; c) H. L. Pan, Y. S. Hu, L. Q. Chen, Energy \& Environmental Science 2013, 6, 2338; d) P.-F. Wang, Y. You, Y.X. Yin, Y.-G. Guo, Advanced Energy Materials 2017, 1701912; e) J.-Y. Hwang, S.-T. Myung, Y.K. Sun, Chemical Society Reviews 2017, 46, 3529.

[4] N. Yabuuchi, M. Kajiyama, J. Iwatate, H. Nishikawa, S. Hitomi, R. Okuyama, R. Usui, Y. Yamada, S. Komaba, Nature materials 2012, 11, 512.

[5] R. Gover, A. Bryan, P. Burns, J. Barker, Solid State lonics 2006, 177, 1495.

[6] a) L. Cailloce, Vol. 2015, 2015; b) S. Roberts, E. Kendrick, Nanotechnology, science and applications 2018, 11, 23.

[7] a) G. Yan, D. Alves-Dalla-Corte, W. Yin, N. Madern, G. Gachot, J.-M. Tarascon, Journal of The Electrochemical Society 2018, 165, A1222; b) G. Yan, R. Dugas, J.-M. Tarascon, Journal of The Electrochemical Society 2018, 165, A220.

[8] a) J. B. Goodenough, Y. Kim, Chemistry of Materials 2010, 22, 587; b) C. Liu, Z. G. Neale, G. Cao, Materials Today 2015, 19, 109; c) D. Aurbach, Y. Talyosef, B. Markovsky, E. Markevich, E. Zinigrad, L. Asraf, J. S. Gnanaraj, H.-J. Kim, Electrochimica Acta 2004, 50, 247.

[9] a) G. G. Eshetu, S. Grugeon, H. Kim, S. Jeong, L. Wu, G. Gachot, S. Laruelle, M. Armand, S. Passerini, ChemSusChem 2016, 9, 462; b) R. Mogensen, D. Brandell, R. Younesi, ACS Energy Letters 2016, 1173.

[10] a) K. Xu, Chemical Reviews 2004, 104, 4303; b) K. Xu, Chem Rev 2014, 114, 11503; c) S. S. Zhang, Journal of Power Sources 2006, 162, 1379; d) D. Aurbach, Journal of Power Sources 2003, 119-121, 497.

[11] a) K. Abe, K. Miyoshi, T. Hattori, Y. Ushigoe, H. Yoshitake, Journal of Power Sources 2008, 184, 449; b) L. Ma, J. Xia, J. R. Dahn, Journal of The Electrochemical Society 2015, 162, A1170; c) L. Madec, R. Petibon, J. Xia, J.-P. Sun, I. G. Hill, J. R. Dahn, 2015, 162, A2635.

[12] a) L. Cheng, R. S. Assary, X. Qu, A. Jain, S. P. Ong, N. N. Rajput, K. Persson, L. A. Curtiss, The Journal of Physical Chemistry Letters 2015, 6, 283; b) P. Jankowski, W. Wieczorek, P. Johansson, Journal of Molecular Modeling 2016, 23, 6; c) Y. Wang, S. Nakamura, K. Tasaki, P. B. Balbuena, Journal of the American Chemical Society 2002, 124, 4408.

[13] a) S. Jurng, Z. L. Brown, J. Kim, B. L. Lucht, Energy \& Environmental Science 2018, 11, 2600; b) S. J. Lee, J. G. Han, I. Park, J. Song, J. Cho, J. S. Kim, N. S. Choi, Journal of the Electrochemical 
Society 2014, 161, A2012; c) L. Zhang, Y. Ma, X. Cheng, P. Zuo, Y. Cui, T. Guan, C. Du, Y. Gao, G. Yin, Solid State Ionics 2014, 263, 146.

[14] a) G. Y. Kim, J. R. Dahn, Journal of the Electrochemical Society 2015, 162, A437; b) Y.-S. Kim, T.-H. Kim, H. Lee, H.-K. Song, Energy \& Environmental Science 2011, 4, 4038.

[15] J. Pires, L. Timperman, A. Castets, J. S. Pena, ErwanDumont, S. Levasseur, R. Dedryvere, C. Tessier, M. Anouti, RSC Advances 2015, 5, 42088.

[16] P. Peljo, H. H. Girault, Energy \& Environmental Science 2018, 11, 2306.

[17] a) J. L. Allen, S.-D. Han, P. D. Boyle, W. A. Henderson, Journal of Power Sources 2011, 196, 9737 ; b) J. Chen, Z. Huang, C. Wang, S. Porter, B. Wang, W. Lie, H. K. Liu, Chemical Communications 2015, 51, 9809.

[18] H. Ota, Y. Sakata, A. Inoue, S. Yamaguchi, Journal of The Electrochemical Society 2004, 151, A1659.

[19] A. Rezqita, M. Sauer, A. Foelske, H. Kronberger, A. Trifonova, Electrochimica Acta 2017, 247, 600.

[20] S. P. Ong, O. Andreussi, Y. Wu, N. Marzari, G. Ceder, Chemistry of Materials 2011, 23, 2979.

[21] D. A. Shirley, Physical Review B 1972, 5, 4709.

[22] J. H. Scofield, Journal of Electron Spectroscopy and Related Phenomena 1976, 8, 129.

[23] a) J. Hutter, M. Iannuzzi, F. Schiffmann, J. VandeVondele, Wiley Interdisciplinary ReviewsComputational Molecular Science 2014, 4, 15; b) J. VandeVondele, M. Krack, F. Mohamed, M. Parrinello, T. Chassaing, J. Hutter, Computer Physics Communications 2005, 167, 103.

[24] a) O. Andreussi, I. Dabo, N. Marzari, Journal of Chemical Physics 2012, 136; b) J. L. Fattebert, F. Gygi, Journal of Computational Chemistry 2002, 23, 662.

[25] Y. Chernyak, Journal of Chemical and Engineering Data 2006, 51, 416.

[26] J. VandeVondele, J. Hutter, Journal of Chemical Physics 2007, 127, 114105.

[27] J. P. Perdew, K. Burke, M. Ernzerhof, Physical Review Letters 1996, 77, 3865.

[28] a) A. D. Becke, The Journal of Chemical Physics 1993, 98, 5648; b) C. Lee, W. Yang, R. G. Parr, Physical Review B 1988, 37, 785.

[29] S. Goedecker, M. Teter, J. Hutter, Physical Review B 1996, 54, 1703.

[30] S. Plimpton, Journal of Computational Physics 1995, 117, 1.

[31] W. L. Jorgensen, D. S. Maxwell, J. Tirado-Rives, Journal of the American Chemical Society 1996, 118, 11225.

[32] S. Grimme, J. Antony, S. Ehrlich, H. Krieg, Journal of Chemical Physics 2010, 132, 154104.

[33] P. Giannozzi, S. Baroni, N. Bonini, M. Calandra, R. Car, C. Cavazzoni, D. Ceresoli, G. L. Chiarotti, M. Cococcioni, I. Dabo, A. Dal Corso, S. de Gironcoli, S. Fabris, G. Fratesi, R. Gebauer, U. Gerstmann, C. Gougoussis, A. Kokalj, M. Lazzeri, L. Martin-Samos, N. Marzari, F. Mauri, R. Mazzarello, S. Paolini, A. Pasquarello, L. Paulatto, C. Sbraccia, S. Scandolo, G. Sclauzero, A. P. Seitsonen, A. Smogunov, P. Umari, R. M. Wentzcovitch, Journal of Physics: Condensed Matter 2009, 21, 395502.

[34] D. Sanchez-Portal, E. Artacho, J. M. Soler, Solid State Communications 1995, 95, 685. 
512 Figure 1 The electrochemical performance of NVPF/C cells using electrolytes with single additives cycled 513 at $55^{\circ} \mathrm{C}$ : a. the voltage-capacity curve with the corresponding $\mathrm{dQ} / \mathrm{dV}$ plot as inset. The positive current 514 shown in the $\mathrm{dQ} / \mathrm{dV}$ plot of the full cells correspond to the reduction process in the hard carbon electrode; 515 b. the voltage-time curves of NVPF/C cell at $11^{\text {th }}$ cycle (self-discharge cycle) and $12^{\text {th }}$ (recovery cycle), and 516 the corresponding $C_{S}\left(Q_{11 t h}-d i s / Q_{11 t h}-c h a\right)$, and $C_{R}\left(Q_{12 t h}-d i s / Q_{11 t h}-c h a\right)$ value; $c-d$. the capacity-retention and direct current resistance evolution during the cycling at $C / 10$ respectively.

Figure 2 The electrochemical performance of NVPF/C cells cycled in the two or three additives added electrolyte at $55 \stackrel{\circ}{\circ}$. a. the $C_{S}$ and $C_{R}$ values at $11^{\text {th }}$ cycle (self-discharge cycle) and $12^{\text {th }}$ (recovery cycle); b-c. the capacity-retention and direct current resistance evolution during the cycling at $C / 10$.

521 Figure 3 The electrochemical performance of NVPF/C cells having electrolytes with four additives with 522 varying amount of $\mathrm{VC}$ cycled at $55^{\circ} \mathrm{C}$. a. the $\mathrm{C}_{\mathrm{S}}$ and $\mathrm{C}_{\mathrm{R}}$ values at $11^{\text {th }}$ cycle (self-discharge cycle) and $12^{\text {th }}$ 523 (recovery cycle); b. the representative voltage-capacity curve at $10^{\text {th }}, 20^{\text {th }}$, and $30^{\text {th }}$ cycle; c-d. the 524 capacity-retention and direct current resistance evolution during the cycling at $C / 10$. Notes: to avoid 525 overwhelm describe the detailed compositions of electrolyte samples, we formatted that one color represents one sample in Fig. $3 \mathrm{a}, \mathrm{b}$, and d. Figure 4 The change in impedance of NVPF/C cells with cycling. The cells were cycled at $55^{\circ} \mathrm{C}$ for 61 cycles and cooled to $25^{\circ} \mathrm{C}$. The EIS data were recorded in discharged state for $1^{\text {st }}, 10^{\text {th }}, 11^{\text {th }}$, and $61^{\text {st }}$ cycle at 55 $529{ }^{\circ} \mathrm{C}$ and at the following $62^{\text {nd }}$ cycle at $25^{\circ} \mathrm{C}$. The Nyquist plots of NVPF/C cells using a. EC-PC electrolyte, b. magic electrolyte. The empty circles show the experimental data and the solid line represents the fitting results using the equivalent circuit model in (d). The red and blue semi-circles in (a) and (b) gives the deconvoluted surface film (Rsf) and charge transfer (Rct) resistance values and their evolution on cycling with different electrolyte formulation are shown in c and e respectively.

Figure 5 The XPS spectra of pristine NVPF and C electrodes, and those cycled electrodes at $1^{\text {st }}, 10^{\text {th }}, 11^{\text {th }}$, and $61^{\text {st }}$ under $55^{\circ} \mathrm{C}$ by using EC-PC or magic A electrolyte. a. the $C_{1 s}$ spectra of NVPF electrode with corresponding atomic ratio underneath; $b$. the $\mathrm{O}_{1 s}$ spectra of NVPF electrode with corresponding atomic ratio underneath; $c$. the $F_{1 s}$ spectra of carbon electrode; $d$. the $P_{2 p}$ spectra of carbon electrode. functional theory based molecular dynamics simulation of the Magic A electrolyte. Energies are represented as box and whisker plots, and are aligned with respected to the Fermi energy of each snapshot. The filled box represents the HOMO energy levels, and the hollow box show the LUMO energy levels. 

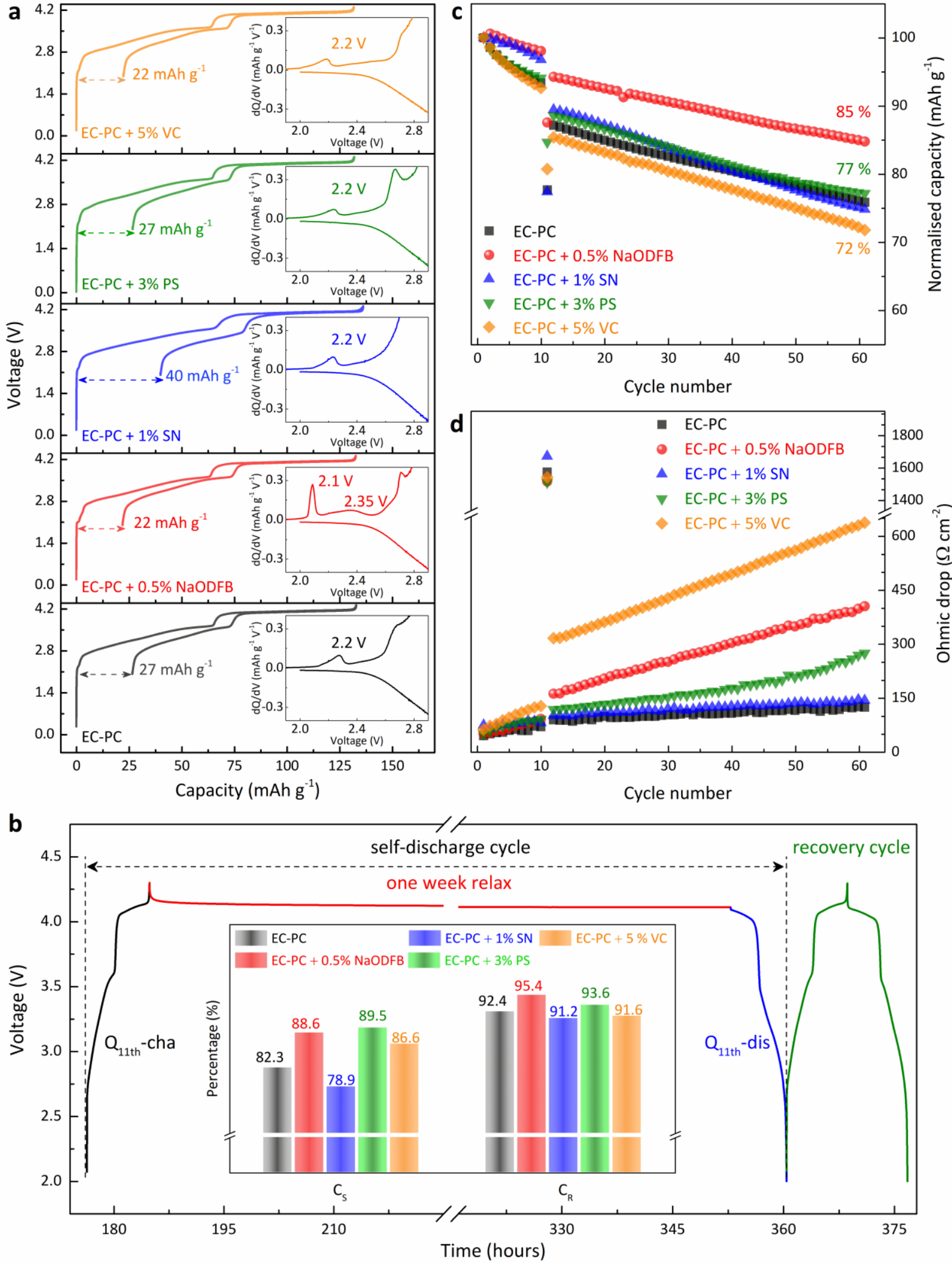
Figure 2
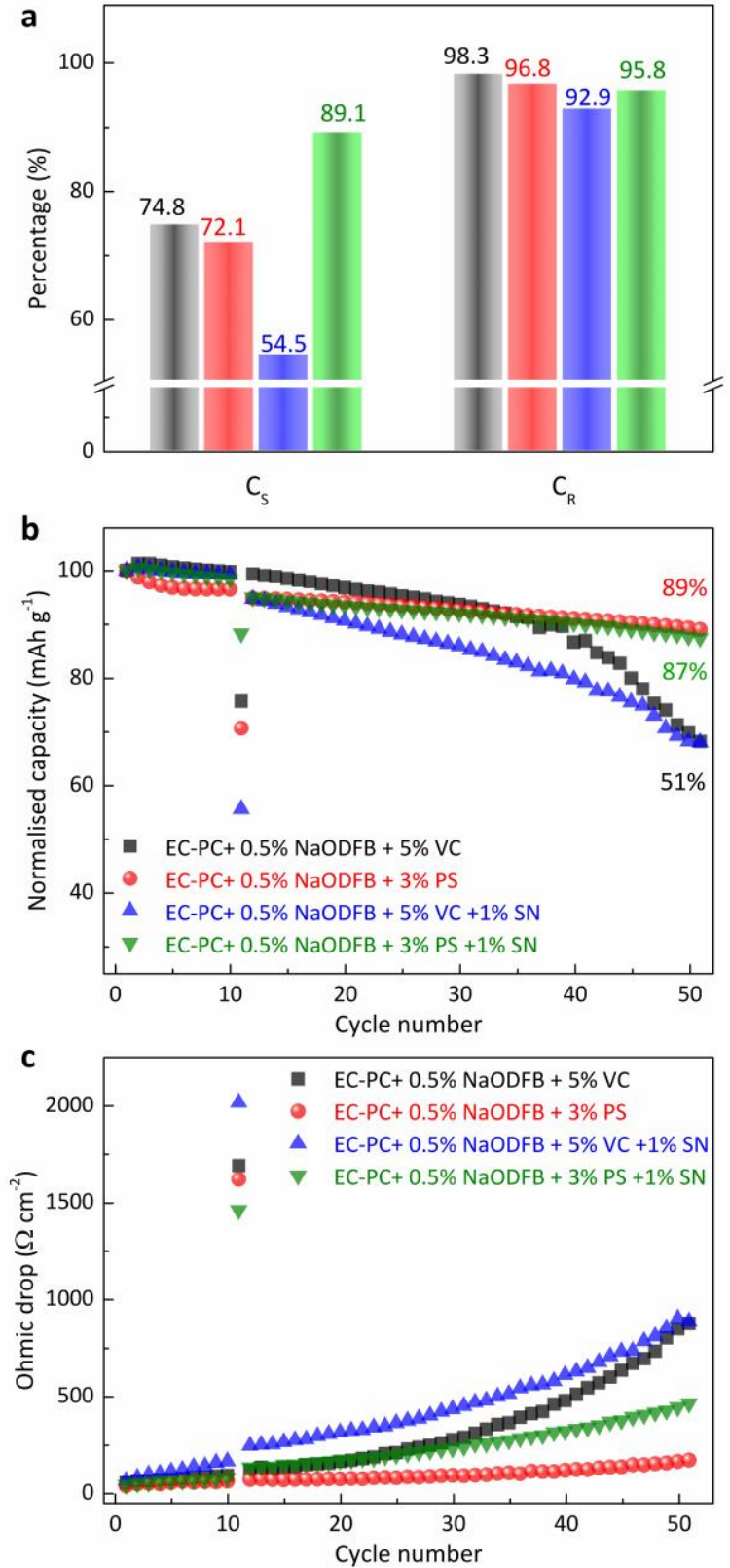
Figure 3
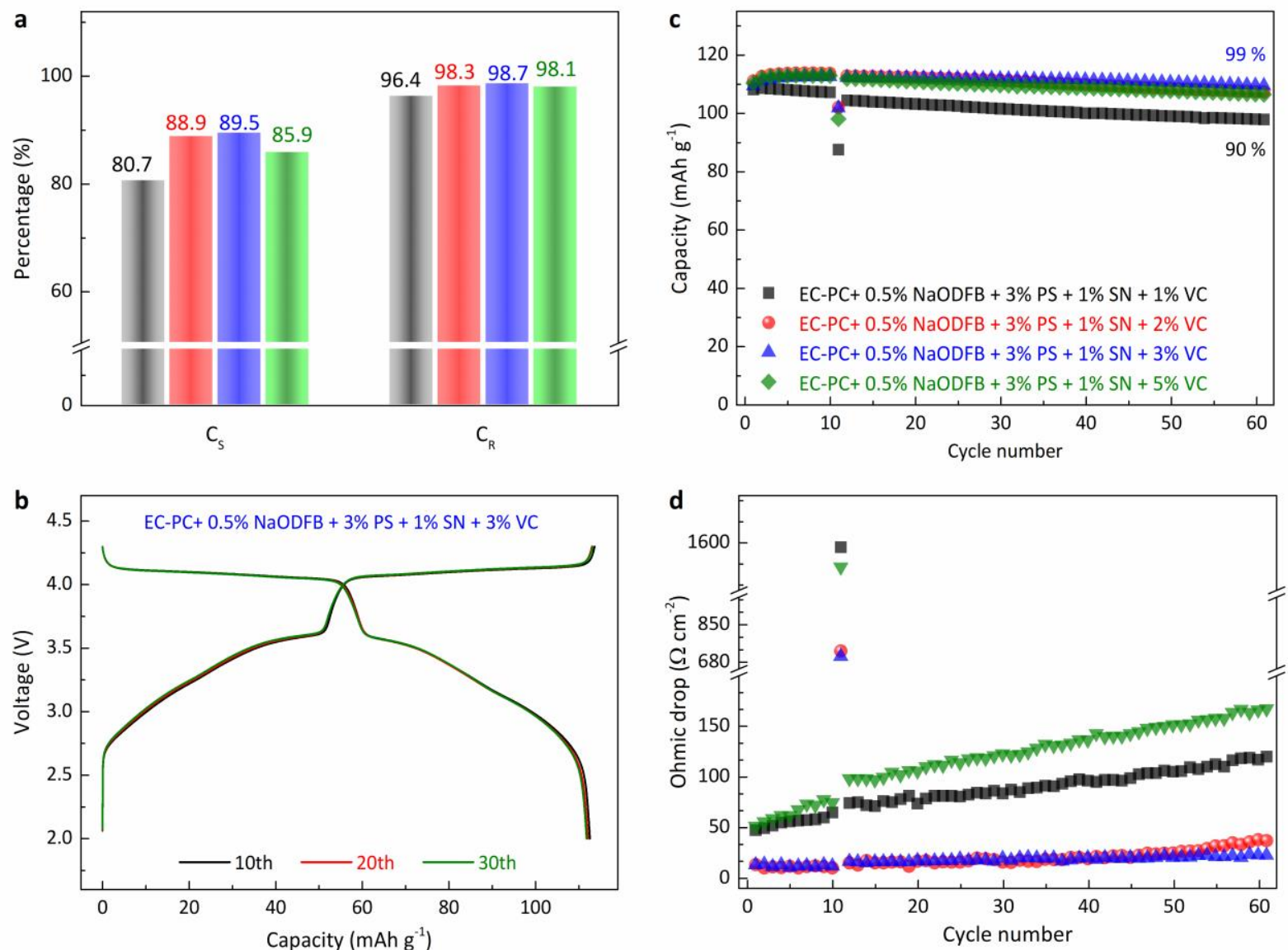

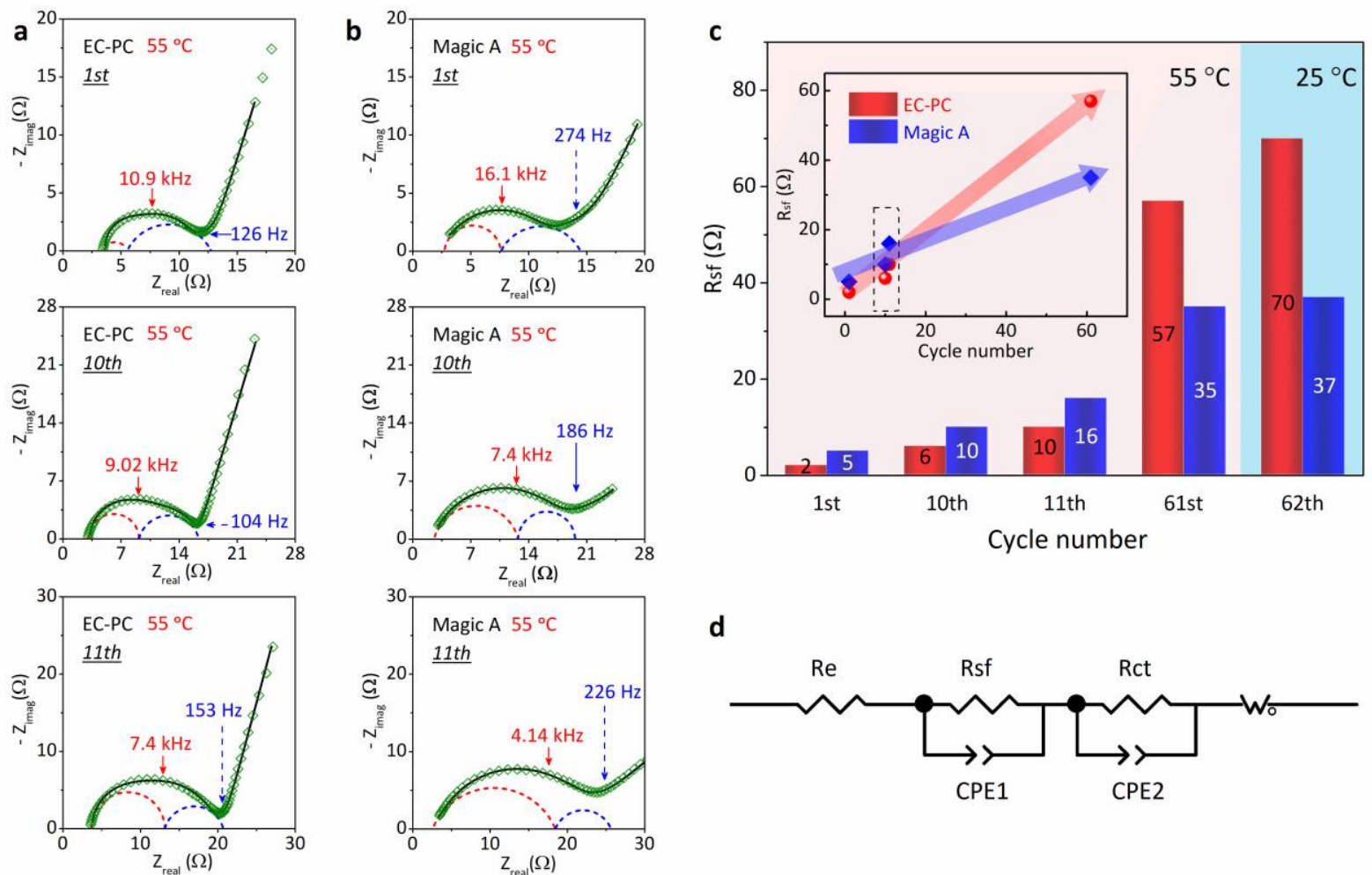

d
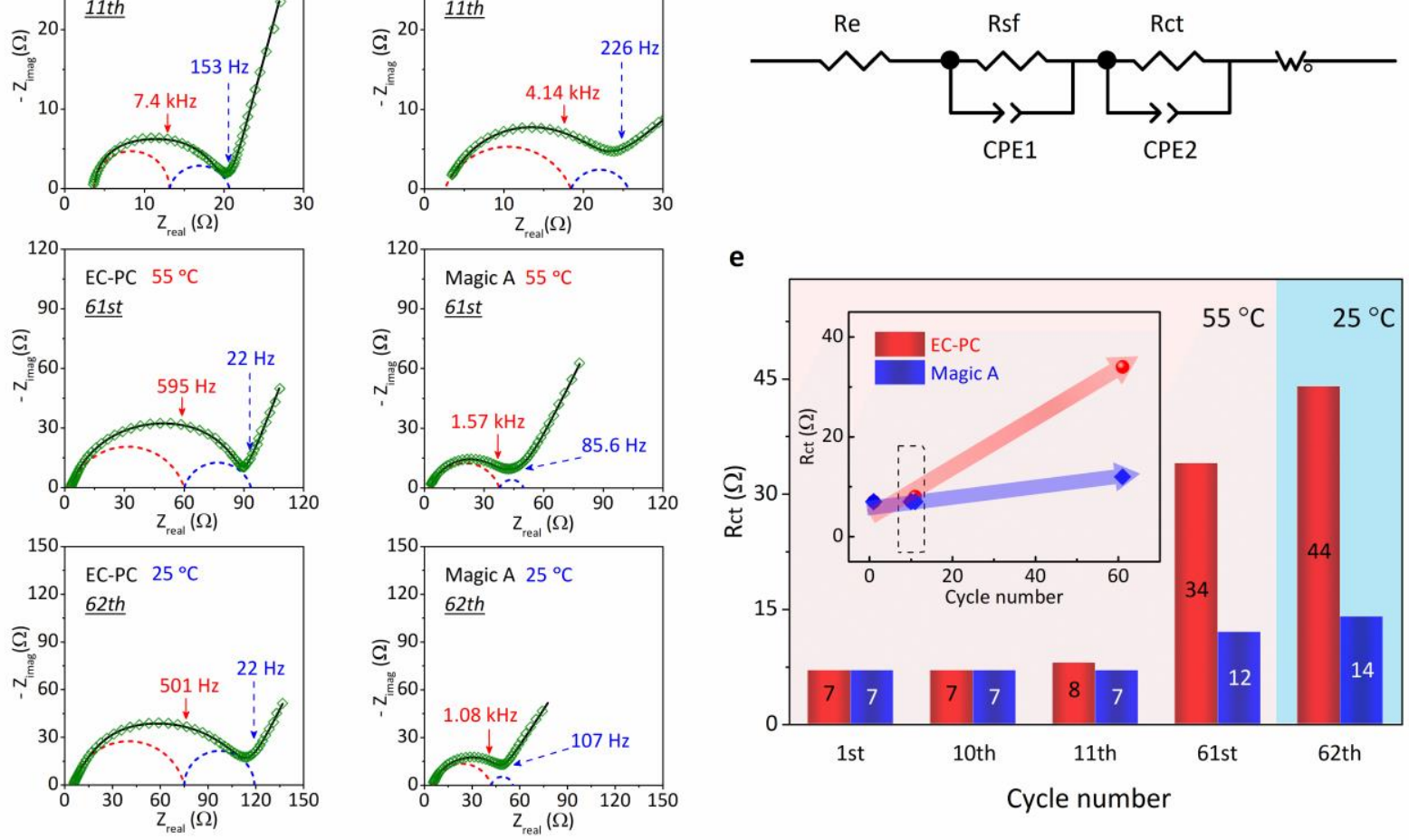

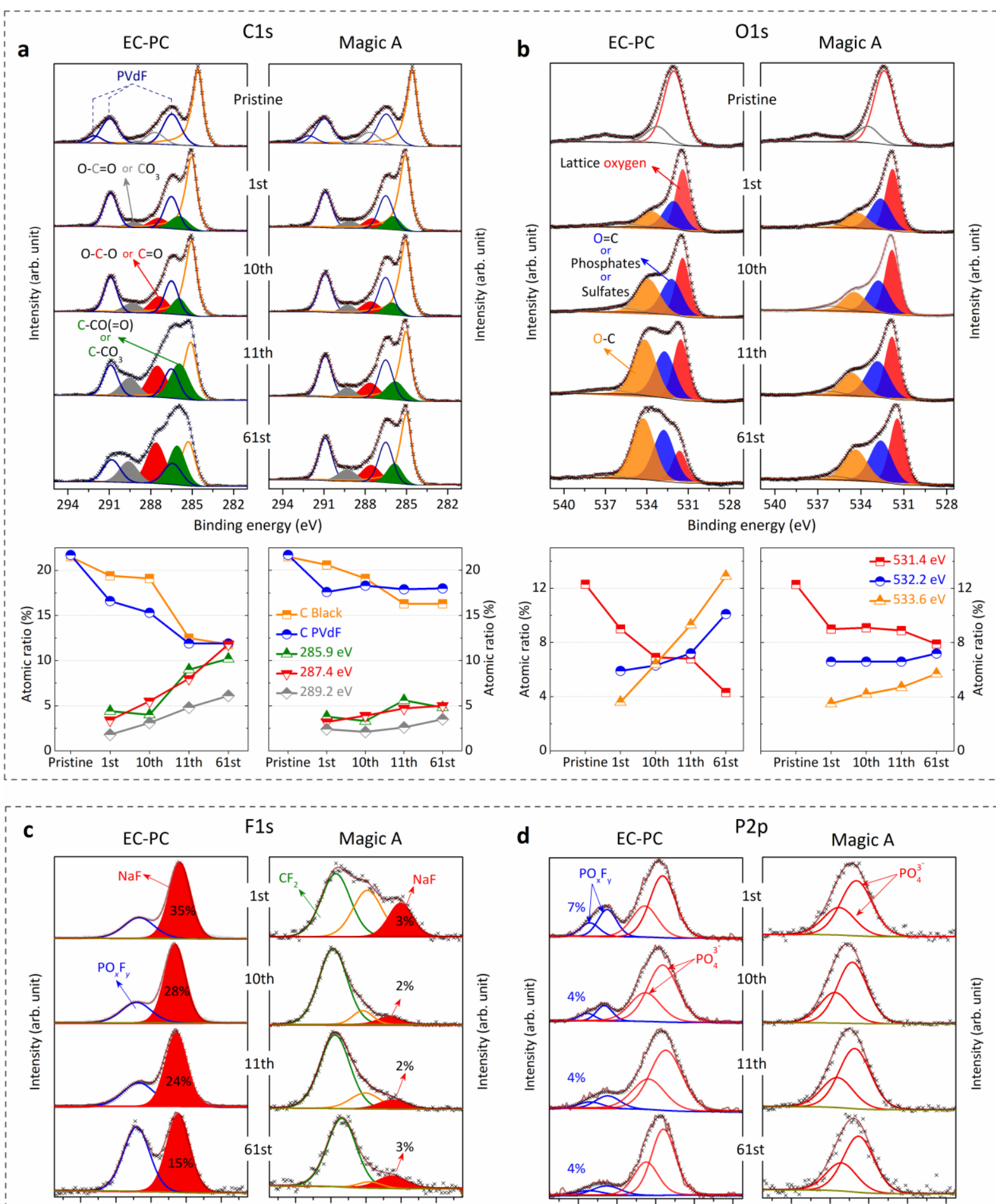

$\begin{array}{llllllllll}690 & 688 & 686 & 684 & 682 & 690 & 688 & 686 & 684 & 682\end{array}$

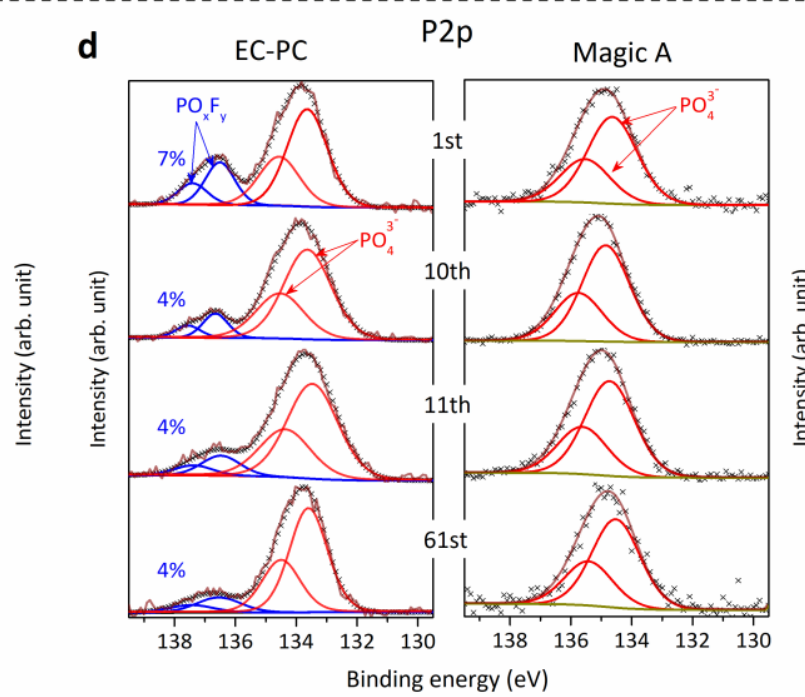


Figure 6

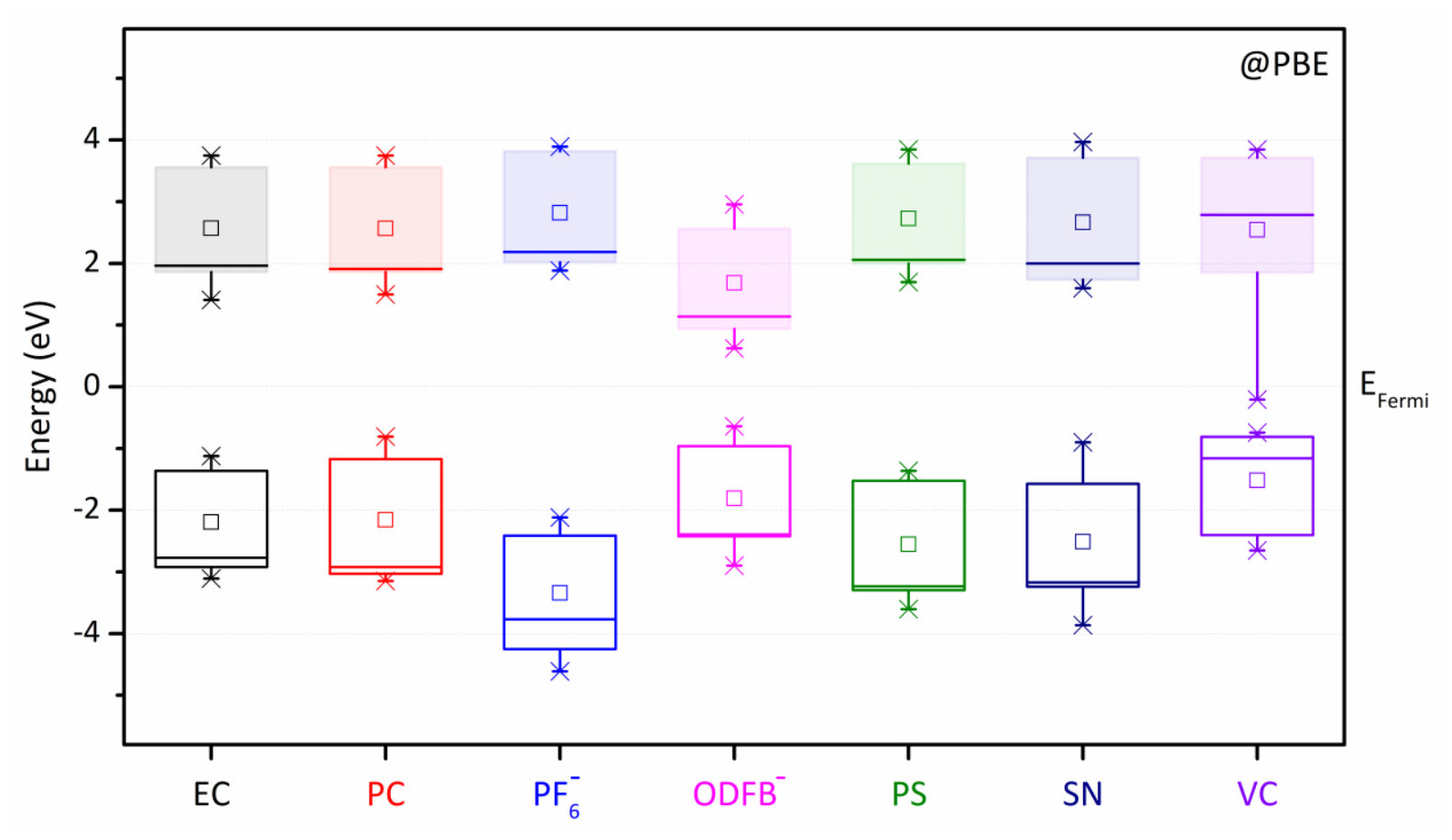

554 